\title{
Dual-responsive shape memory polymer arrays with smart and precise multiple-wetting controllability
}

\author{
Dongjie Zhang ${ }^{1}$, Qixing Xia ${ }^{2}$, Hua Lai ${ }^{1}$, Zhongjun Cheng ${ }^{1 *}$, Pengchang Liu ${ }^{1}$, Haiyang Zhang ${ }^{1}$, \\ Yuyan Liu ${ }^{1 *}$ and Lei Jiang ${ }^{3}$
}

\begin{abstract}
Smart-controlled surface wettability from superhydrophilicity to superhydrophobicity has been extensively explored, and stimulus-responsive strategies have been widely accepted as a useful method to realize reversibility. However, achieving smart and precise wetting control remains challenging because most previous studies focused on stimulating single surface chemistry or microstructures. Herein, a dualstimulus-responsive strategy that can synergistically stimulate surface chemistry and microstructures is demonstrated on the pH-responsive molecule poly(2-(diisopropylamino)ethyl methacrylate (PDPAEMA)-modified temperature-triggered shape memory polymer (SMP) arrays. The responsive PDPAEMA and SMP can provide the surface with tunable surface chemistry and microstructures, respectively. Thus, the wetting of the surface between various states can be reversibly and precisely controlled from superhydrophilicity to superhydrophobicity with contact angle (CA) differences of less than $15^{\circ}$ under the cooperative effect between the adjustable surface microstructure and chemistry. The surface is further utilized as a platform to create gradient wettings based on its excellent controllability. Therefore, this work presents a strategy for surface wetting control by combining tunable surface microstructures and chemistry. The prepared samples with a special wetting controllability can be applied to numerous fields, including adaptive liquid microlenses, accurate drug release, and selective catalysis. This work also proposes novel expectations in designing smart functional surfaces.
\end{abstract}

Keywords: wetting control, PDPAEMA, shape memory polymer, superhydrophobicity, superhydrophilicity

\section{INTRODUCTION}

The smart control of water wetting performances on solid surfaces has been extensively explored because of its potential for application in many fields [1-5], such as controllable cell and protein adhesion [6-8], smart droplet manipulation in microfluidics $[9,10]$, selective oil/water separation [11-13], and accurate drug delivery [14]. Stimulus-responsive strategies have been widely accepted as a useful way to achieve reversible wetting control, and the most common examples are rough structures modified with stimulus-responsive molecules, such as temperatureresponsive polymer poly( $N$-isopropylacrylamide) [15], $\mathrm{pH}$-responsive carboxylic acids [16,17], and other materials with responsivity to ions $[18,19]$, gas [20-22], electrons [23-25], light [26-28], magnetic fields [29,30], and other stimuli. Noticeably, on modified responsive molecule surfaces, surface micromorphology is constant, so it can only provide an invariable surface roughness and a fixed enhanced effect [15-17]. As a result, wetting controllability is limited when the surface chemical composition is changed (superhydrophobicity/superhydrophilicity on rough surfaces and hydrophobicity/hydrophilicity on smooth surfaces). In addition to surface chemistry, the external stimuli of surface microstructures can be subjected to reversible wetting control; the most common examples are elastic polydimethylsiloxane (PDMS) and shape memory polymers (SMPs) with tunable pillar structures under external stretching [31,32], curving [33], magnetic driving [34], and pressing [35]. As for surfaces with tunable microstructures, a narrow transition range can be observed because surface chemical

\footnotetext{
${ }^{1}$ State Key Laboratory of Urban Water Resource \& Environment, School of Chemistry and Chemical Engineering, Harbin Institute of Technology, Harbin 150001, China

${ }^{2}$ Institute of Culture and Heritage, Northwest Polytechnical University, Xi'an 710000, China

${ }^{3}$ CAS Key Laboratory of Bio-Inspired Materials and Interfacial Science, Technical Institute of Physics and Chemistry, Chinese Academy of Sciences, Beijing 100190, China

* Corresponding authors (emails: chengzhongjun@iccas.ac.cn (Cheng Z); liuyy@hit.edu.cn (Liu Y))
} 
compositions are invariable and constantly hydrophobic (i.e., transition from superhydrophobicity to hydrophobicity can occur when surface microstructures are changed) [31]. Therefore, precise wetting control, that is, multiple wetting states with a limited contact angle (CA) difference from superhydrophobicity to superhydrophilicity on the same surface, remains a challenge by singly stimulating surface chemistry or microstructure although smart wetting control can be achieved.

Precise wetting control is significant in many applications, such as accurate drug release, adaptive liquid microlenses, and controllable immiscible liquid separation. For instance, by modifying an electrospun fiber textile with various functional groups, Wang et al. [36] accurately controlled surface wettability and obtained a series of films with different wettabilities. Based on these films, several immiscible organic liquids in mixtures have been successfully separated with high efficiency. Similarly, by precisely controlling the wettability of an electrospun fiber textile, Grinstaff's group [14] reported a tunable and accurate drug release based on these materials. However, precise wetting control can only be observed on different surfaces because all surface microstructures are fixed and only surface chemistry is varied, and each surface can only display a constant wetting performance, which lacks smart controllability. Although the integration of smart wetting and precise controllability is promising to overcome such imperfections and provide surfaces with much better wetting performances, intelligence, and controllability to meet realistic complex requirements, related research is limited.

In this study, a dual-stimulus-responsive strategy is proposed, and the smart and precise control of wetting under the synergistic effect of surface chemical compositions and microstructures is achieved (Fig. 1a). A new surface is prepared by grafting poly(2-(diisopropylamino) ethyl methacrylate) (PDPAEMA) with $\mathrm{pH}$ responsivity onto a temperature-triggered pillar-structured SMP. PDPAEMA and SMP can provide the surface with responsive surface chemistry and microstructures, respectively. Therefore, different surface wettabilities from superhydrophobicity to superhydrophilicity with CA differences of less than $15^{\circ}$ can be reversibly and precisely regulated on the surface by simultaneously controlling water $\mathrm{pH}$ and pillar height $(H)$. Although some dual- or even multiple-responsive superwetting surfaces have been reported [37,38], previous studies focused on singly stimulating surface chemistry based on different external stimuli. In contrast to previously described strategies, our dual-stimulus-responsive strategy based on the co- ordinated regulation of the surface chemical composition and microstructure can provide some novel concepts for creating functional surfaces with tunable wettability and overcome the drawbacks of the studies on precise wetting control. Furthermore, given the advantages of the asprepared surface, it can be used for various applications, including controllable friction, accurate drug release, and selective catalysis.

\section{EXPERIMENTAL SECTION}

\section{Materials}

Before being used, a DPAEMA (99\%, Sigma-Aldrich) monomer was purified by passing through DHR-4, an inhibitor-removing column. Diglycidyl ether of bisphenol A-type epoxy resin (DGEBA, Bluestar Chemical New Materials Co.), $m$-xylylenediamine (MXDA, Changsha Institute of Chemical Industry), $n$-octylamine (OA, J\&K Scientific), trichloro $(1 H, 1 H, 2 H, 2 H$-perfluorooctyl)silane (97\%, Sigma-Aldrich), (3-aminopropyl) triethoxysilane

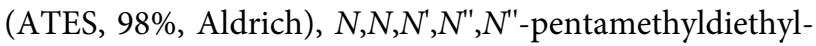
enetriamine (99\%, Aldrich), 2-bromoisobutyryl bromide (98\%, Aldric), and copper(I) bromide (99.999\%, Aldrich) were directly utilized without further modifications or processing.

\section{Fabrication of SMP surface}

An SMP surface was fabricated via template transferring. First, a micropillar-structured silicon mold was obtained through photolithography. Then, a PDMS template was prepared by duplicating the structured silicon template. Afterward, a prepolymer containing DGEBA, MXDA, and OA (molar ratio of 8:3:2) was transferred into the PDMS mold. After the system was sequentially heated at $40^{\circ} \mathrm{C}$ for $1 \mathrm{~h}, 60^{\circ} \mathrm{C}$ for $2 \mathrm{~h}$, and $100^{\circ} \mathrm{C}$ for $10 \mathrm{~h}$ in an oven, the SMP surface could be obtained by carefully peeling. The fabrication process is further described in detail in Figs S1 and S2, and the related description is presented in the Supplementary information.

\section{Preparation of PDPAEMA-grafted SMP surface}

SMP was initially bombarded with $\mathrm{O}_{2}$ plasma for $5 \mathrm{~min}$ to generate surface hydroxyl groups. Afterward, the SMP surface was treated with $95 \%$ ethanol containing $5 \%$ ATES $(v / v)$ and a certain amount of glacial acetic acid (with solution $\mathrm{pH}$ adjusted to about 5.5) at room temperature to prepare an amino group graft to the SMP surface. After $2 \mathrm{~h}$, the SMP substrate was rinsed with an excess of ethanol and dried under a nitrogen flow. Subsequently, the SMP substrate was immersed in dry hexane 

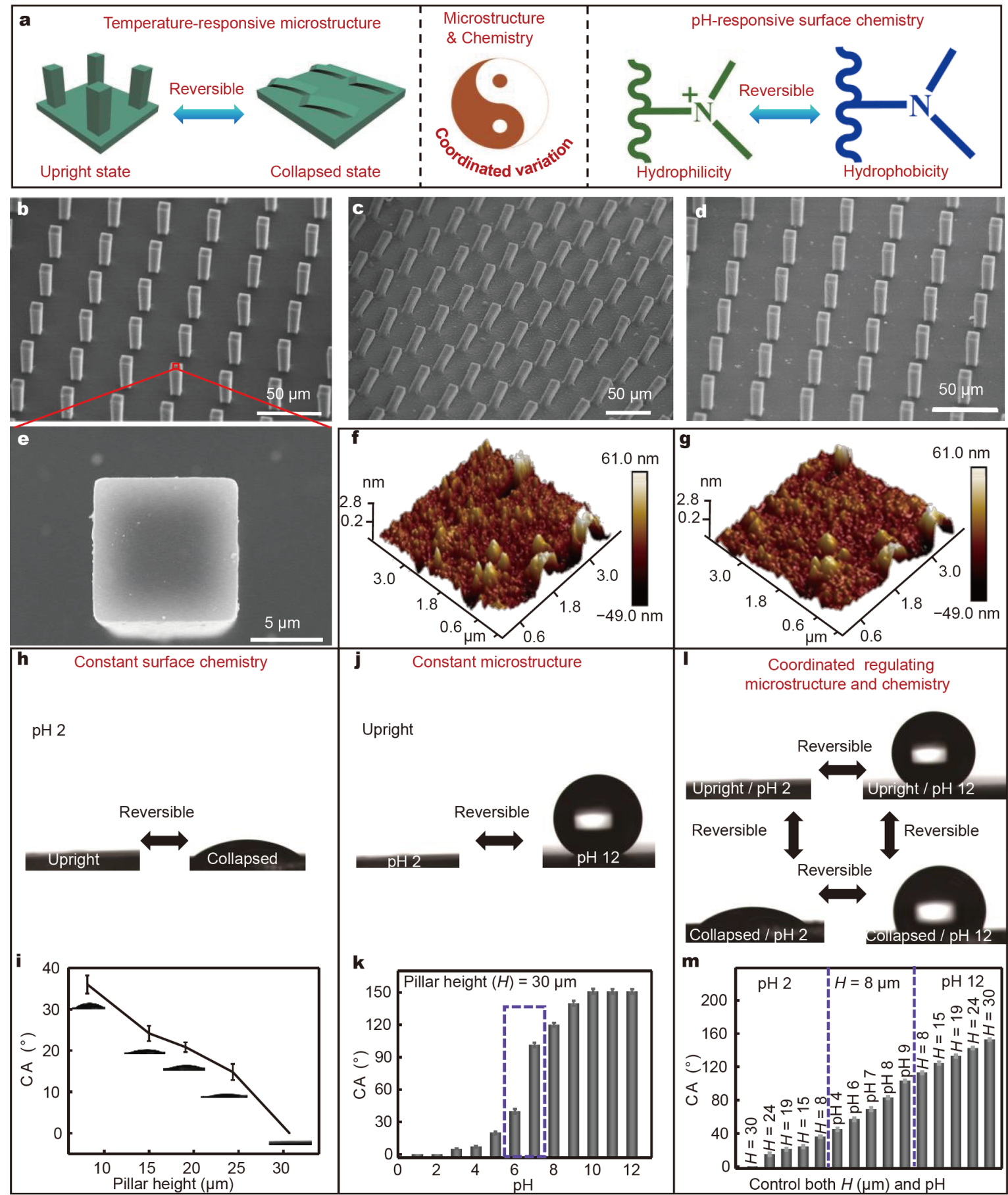

Figure 1 (a) Illustration of the advanced dual-responsive strategy for wetting control based on the synergistic regulation of the temperatureresponsive shape memory microstructure and the pH-responsive surface chemistry. (b-d) Scanning electron microscopy (SEM) images of PDPAEMA-modified SMP pillars at original upright, collapsed, and recovered states, respectively. (e) The magnified image of one pillar. (f, g) 3D morphologies of the pillar tips corresponding to (b) and (d) tested via AFM. PDPAEMA-grafted surfaces retain a remarkable shape memory property. (h) CA results with $\mathrm{pH} 2$ on the surface with upright pillars (left) and collapsed pillars (right) limit the transition from general hydrophilicity to superhydrophilicity by singly changing the pillar height (i). (j) Shapes of water droplets with pH 2 (left) and 12 (right) on the surface with an upright pillar structure. Only the surface chemistry can be controlled by singly changing water $\mathrm{pH}$, and a large CA difference is inevitable (k). (l) Water droplet outlines on the surface under the regulation of both surface microstructure and chemistry, indicating that smart multiple wetting control can be achieved precisely with a CA difference of less than $15^{\circ}(\mathrm{m})$. 
containing pyridine $(1.6 \%, v / v)$. After $10 \mathrm{~min}$ of precooling, 2-bromoisobutyryl bromide $(2.6 \%, v / v)$ was mixed into the solution. The mixture was stored at $0^{\circ} \mathrm{C}$ for $1 \mathrm{~h}$ and then room temperature for $12 \mathrm{~h}$. The SMP substrate grafted with the initiator was repeatedly washed with hexane and ethanol and dried under a nitrogen flow. The SMP substrate was immersed into degassed $\mathrm{CH}_{3} \mathrm{OH}$, which contained DPAEMA $(1500 \mu \mathrm{L})$ and copper(I) bromide $(0.064 \mathrm{~g}, 0.46 \mathrm{~mol})$. The reaction was continued for $12 \mathrm{~h}$ at room temperature under the nitrogen flow. Lastly, the SMP substrate was cleaned with copious ethanol and water and dried under the nitrogen flow.

\section{Control of the surface wettability by tuning surface chemical composition and microstructure}

The solutions with various $\mathrm{pH}$ levels were prepared as follows. The solutions with extreme $\mathrm{pH}$ levels ranging in 1-4 and 9-12 were obtained with aqueous solutions of $\mathrm{HCl}$ and $\mathrm{NaOH}$. The solutions with $\mathrm{pH}$ levels from 5 to 8 were prepared with phosphate-buffered saline $\left(\mathrm{Na}_{2} \mathrm{HPO}_{4} /\right.$ $\mathrm{NaH}_{2} \mathrm{PO}_{4}$ ).

The samples were pressed at a certain pressure $(\sim 2 \mathrm{MPa})$ for $10 \mathrm{~min}$ at temperature above the glass transition temperature $\left(T_{\mathrm{g}}\right)$ of SMP (about $125^{\circ} \mathrm{C}$ ) and cooled down to room temperature under a presser to obtain variations in the microstructure. Afterward, the recovery time was controlled, and vatiations with different heights of pillars were prepared. The CAs of droplets with different $\mathrm{pH}$ levels were tested. The samples were cleaned with profuse water and methanol and dried under $\mathrm{N}_{2}$ flow between CA measurements with droplets of various $\mathrm{pH}$ values to ensure that the droplets of each $\mathrm{pH}$ level would not interact with other droplets with different $\mathrm{pH}$ levels on the same substrate.

\section{Characterization}

A field-emission scanning electron microscope (SEM, SU8010, HITACHI) and an atomic force microscope (AFM, Dimension Icon, Bruker) were used to measure the surface morphologies. A CA meter (JC 2000D5, Shanghai Zhongchen Digital Technology Apparatus Co., Ltd.) was employed to test water CA. A laser scanning confocal microscope (LSCM, OLS4100, Olympus) was utilized to measure the three-dimensional (3D) microstructure and corresponding profile curves. A Mettler SDTA861e analyzer was used to measure the dynamic mechanical analysis data including the loss tangent $(\tan \theta)$ and storage modulus of the specimens. A tensile tester (Instron 5965) was applied to measure the mechanical strength of materials. A TA Q800 dynamic mechanical analyzer (USA) was used to obtain the shape memory properties of the samples. Elementary analysis was conducted through X-ray photoelectron spectroscopy (XPS, PHI 5400 ESCA System). An optical microscope (XPR$700 \mathrm{~V}$ ) with a hot stage was employed to observe the pillar state in situ during recovery. A variable angle spectroscopic ellipsometer (VASE, J.A. Woollam RC2) was used to test the thickness of PDPAEMA.

\section{RESULTS AND DISCUSSION}

SMPs have been widely explored because of their extraordinary shape memory effect and widespread applications, including smart adhesives [39,40], optical chips [41,42], and biomedical devices [43]. Their wetting control has been rarely investigated and only recently reported; similar to elastic PDMS [31], SMPs have a limited wetting transition in a finite range [35,44-47]. In our work, this problem can be easily solved by simply grafting a responsive molecule onto SMP. First, the microstructured SMP sample is prepared by polymerizing DGEBA, MXDA, and OA on a microstructured silicon mold (Figs S1-S3) [48]. Fig. S4 displays the SEM image of the as-prepared SMP surface viewed at a tilt angle of about $40^{\circ}$. Regular pillars with a diameter, spacing, and height of about 10,30 , and $30 \mu \mathrm{m}$, respectively, are uniformly distributed on the surface. The top of the pillar is smooth (the inset of Fig. S4), which is similar to the silicon template (Fig. S3). AFM imaging further confirms the result and demonstrates that the surface roughness of the pillar tip is as low as $0.52 \mathrm{~nm}$ (Fig. S5). The pillars are initially pressed with a flat glass slide under an external force at $125^{\circ} \mathrm{C}$ (higher than the polymer's $T_{\mathrm{g}}$ of about $98.8^{\circ} \mathrm{C}$; Fig. S6a) to test the shape memory property of the surface. The surface is reheated to investigate the recovery property (Fig. S7). All the pillars collapse after they are pressed, and they spontaneously return to their original upright form after they are reheated (Fig. S4). This result indicates that the as-prepared samples have a remarkable shape memory property. The as-prepared SMP arrays are further modified with PDPAEMA through atom-transfer radical polymerization (ATRP) [49], and they can be demonstrated by the XPS results (Fig. S8). The thickness of the PDPAEMA coating is about $16.5 \mathrm{~nm}$. Herein, the substrates with the same diameter $(10 \mu \mathrm{m})$ and height $(30 \mu \mathrm{m})$ of the pillars with different spacings $(5,10,20$, and $30 \mu \mathrm{m}$, respectively) are modified with the same PDPAEMA to obtain the surface with the best $\mathrm{pH}$-responsive wetting controllability. The results show that the sample with $30 \mu \mathrm{m}$ spacing has the best controllability, so it is selected as the surface in the subsequent analysis 
(Fig. S9). Fig. 1b-d display the SEM results of the samples modified with PDPAEMA at original upright, collapsed, and recovered states, respectively. The pillars' dimension and morphology have no apparent differences from those on the original SMP surface, indicating that the pillar structures can still be kept after ATRP. Noticeably, after the modification with PDPAEMA, the pillar tips become rougher (Fig. 1e). The AFM result reveals that the surface roughness of the pillar tips increases to about $9.56 \mathrm{~nm}$ (Fig. 1f), demonstrating that some nanostructures form on the microscale pillars, which are similar to microstructures on superwetting biological surfaces in nature [50]. Similar to the original SMP surface, the PDPAEMAmodified surface retains its excellent shape memory effect. As shown in Fig. 1c, d, after being pressed and reheated, the pillars can exhibit the collapsed and upright states, respectively. After the recovery, the hierarchical micro/nanostructure can still be observed, and the surface roughness on the pillar tips is similar to that before the specimens are pressed (about $9.17 \mathrm{~nm}$, Fig. 1g). Therefore, the modification of PDPAEMA on the pillar-structured SMP can result in the formation of micro/ nanostructures on the surface, and the excellent shape memory effect of the obtained surface is similar to that of the original SMP surface.

The $\mathrm{pH}$-responsive PDPAEMA-modified SMP arrays retain their shape memory effect. This result indicates that the surface microstructure and chemistry can be regulated under external stimuli on the as-prepared surface. For a water droplet with a given $\mathrm{pH}$ (e.g., water with $\mathrm{pH} 2$ ), the CA can be reversibly tuned between $0^{\circ}$ and $36^{\circ}$ if the surface microstructure between the upright and collapsed states is changed (Fig. 1h). Even when the surface microstructure is controlled more precisely (herein, the microstructure is controlled by changing the pillar's height), the variation in the CA is still restricted in a limited hydrophilic region (Fig. 1i). A similar controllability can also be observed in a basic water droplet, and the only difference is that the variation is in the hydrophobic region (Fig. S10). These results indicate that the CA slightly varies when the surface microstructure is individually regulated. Furthermore, transition from hydrophilicity to hydrophobicity is impossible. In contrast to singly regulated surface microstructures, singly regulated surface chemistry can provide a broad tunability to surfaces. For example, in Fig. 1j, water droplets with different $\mathrm{pH}$ levels are used as the stimuli for surface chemistry because PDPAEMA is responsive to water $\mathrm{pH}$. The surface with an upright pillar structure exhibits a reversible superhydrophilicity/superhydrophobicity tran- sition as the water $\mathrm{pH}$ varies between 2 and 12 . When the water $\mathrm{pH}$ is changed more precisely (Fig. $1 \mathrm{k}$ ), more wetting states from superhydrophilicity (CA of nearly $0^{\circ}$ ) to superhydrophobicity (CA of about $153^{\circ}$ ) can be obtained. However, a sudden transition from hydrophilicity to hydrophobicity with a large CA difference (about $60^{\circ}$ ) is difficult to be avoided (emphasized by the dotted box). Therefore, accurately controlling the wetting property is difficult. This defect is inevitable on all reported PDPAEMA-modified static micro/nanostructured surfaces; consequently, their application in numerous fields, such as subtly tunable cell/tissue growth and precise drug release, is limited [20-25,51]. Although the sudden transition of the CA (large CA difference) can be eliminated in collapsed pillars, the surfaces lose their superhydrophilicity/superhydrophobicity, and the wetting control range decreases (Fig. S11). These results reveal that numerous imperfections, including limited variation range and large CA difference, are still ineluctable by singly regulating the surface chemistry or the microstructure. With our dual-stimulus-responsive strategy, all these problems can be resolved, and smart and precise wetting control between multiple wetting states can be achieved. As shown in Fig. 11, in addition to the transitions caused by single surface chemistry or microstructural variations (e.g., superhydrophilicity/ superhydrophobicity, hydrophilicity/hydrophobicity transitions due to surface chemistry variation, superhydrophilicity/hydrophilicity and superhydrophobicity/ hydrophobicity transitions caused by surface microstructure variation), some new phenomena, such as transition between superhydrophilicity and general hydrophobicity (upper left and bottom right in Fig. 1l) or transition from superhydrophobicity to general hydrophilicity, can be achieved (upper right and bottom left in Fig. 11) by simultaneously adjusting the $\mathrm{pH}$ of water and the morphological characteristics of the pillars. These findings demonstrate that the wetting performance of surfaces can be adjusted from one random state to another in a wide range between superhydrophobicity and superhydrophilicity. Different wetting states from superhydrophilicity to superhydrophobicity with a CA difference of less than $15^{\circ}$ can be gradually and precisely controlled (Fig. $1 \mathrm{~m}$ ). To the best of our knowledge, such high-precision wetting control is yet to be reported. These results indicate that our dual-stimulus-responsive strategy can provide surfaces with better wetting performance and controllability than those of traditional single-stimulusresponsive strategies that singly control surface chemical compositions or microstructures. Furthermore, great re- 
sponsivity by the governing surface chemistry (Fig. 2a), surface micromorphology (Fig. 2b), or both (Fig. 2c, d) remain in all the above transitions after repeating several times. These transitions can still be observed even after about a month without any special protection. Therefore, our surface has good controllability and stability.

The proposed dual-stimulus-responsive strategy is based on the coordinated effect between responsive surface microstructures and chemical compositions. The changes in the surface microstructure is investigated with LSCM to better understand controllability. Fig. 3a, b show the 3D LSCM results and the corresponding profile pictures of the sample, demonstrating that the size and morphology of the pillars are consistent with the SEM result. When the external force is employed to press the pillars, all of them on the surface collapse (Fig. 3c). The outline shows that the average height of the pillar decreases sharply to about $8 \mu \mathrm{m}$ (Fig. 3d). When the surface is further reheated at $90^{\circ} \mathrm{C}$ for about $128 \mathrm{~s}\left(T_{\mathrm{g}}\right.$ of the asprepared surface is about $84^{\circ} \mathrm{C}$, Fig. S6b), the pillars can spontaneously recover their original shapes and heights (Fig. 3e, f). This result further confirms that the surface has a great shape memory ability. Meanwhile, the samples demonstrate a good fatigue durability (Fig. S12) because of the inherent $3 \mathrm{D}$ crosslinking networks of SMP, and the pillars can be cycled at different states. In Fig. 3g, after several pressing/recovery cycles, the average pillar height does not apparently decrease. Moreover, the recovery process relies on heating time and temperature. The required time for microstructure restoration shortens as the heating temperature increases. The recovery of the original shape needs about $128 \mathrm{~s}$ at $90^{\circ} \mathrm{C}$, but it decreases to about $26 \mathrm{~s}$ at $140^{\circ} \mathrm{C}$ (Fig. 3h), possibly because of the improved chain motility at higher temperatures [47]. When the heating temperature is set at a relatively low value $\left(90^{\circ} \mathrm{C}\right)$, surfaces with various pillar heights can be obtained by simply controlling the heating time (Fig. 3i). Therefore, the surface with different pillar heights and corresponding wetting performances can be observed (Fig. 1i, m).

The variation in surface chemical compositions is investigated through XPS. Fig. 4a displays the XPS results of the surface in the pristine state and after acid and base treatments. The pristine surface shows N 1s peaks at 399 and $401 \mathrm{eV}$, corresponding to the tertiary and quaternary amines of PDPAEMA, respectively [52]. The amount of quaternary amine is extremely low (about 4.36\%, Fig. 4b). After the treatment with the acidic solution, a new $\mathrm{Cl} 2 \mathrm{p}$
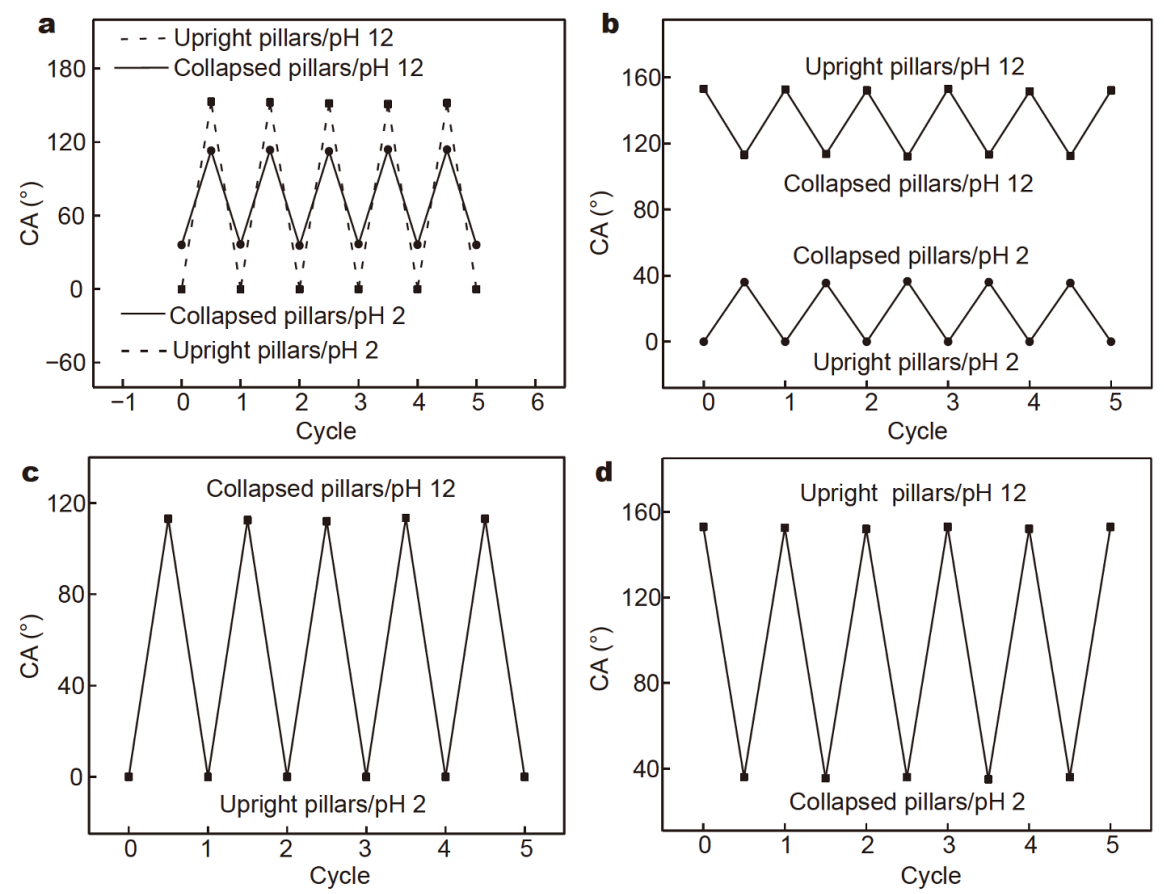

Figure 2 Repeated variation in the surface between different wetting states. (a) Transitions between superhydrophilicity/superhydrophobicity (dotted line) and hydrophilicity/hydrophobicity (solid line) by singly controlling the surface chemistry. (b) Transitions between superhydrophobicity/ hydrophobicity (upper line) and superhydrophilicity/hydrophilicity (bottom line) by singly controlling the surface microstructure. (c, d) Transitions between hydrophobicity/superhydrophilicity and superhydrophobicity/hydrophilicity by synergistically adjusting the surface chemical composition and microstructure, respectively. 

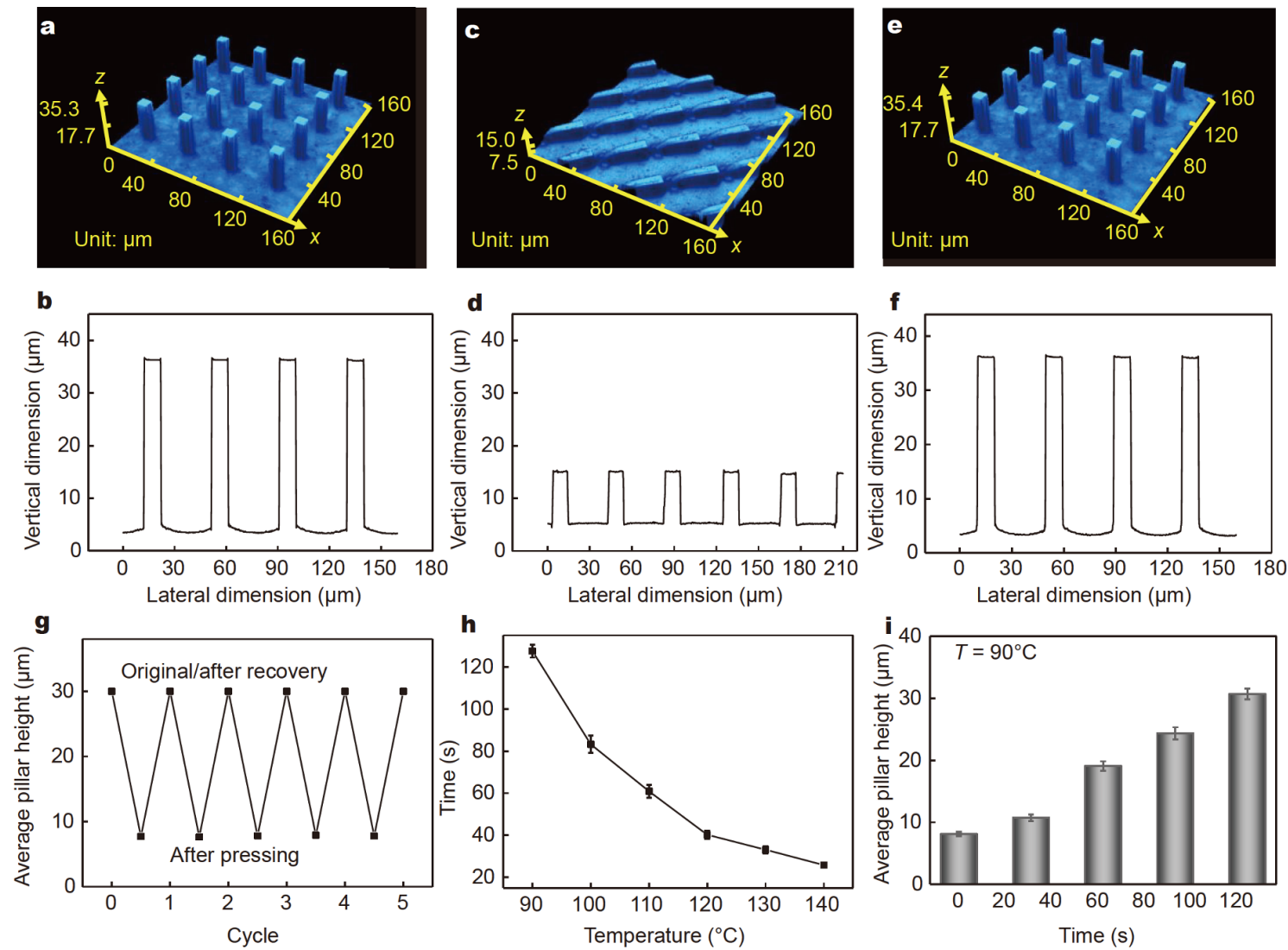

Figure 3 3D confocal images of the surface modified with PDPAEMA and corresponding outline pictures: (a, b) original structure, (c, d) collapsed structure, and (e, f) recovery structure. (g) The average height of the pillar on the surface after several continuous pressing/recovery cycles. (h) Relationship of heating temperature and recovery time. (i) Statistical analysis of the average pillar height on the surface after heating for different durations at $90^{\circ} \mathrm{C}$, indicating that the surface can memorize and display different pillar heights.

peak appears (orange line in Fig. 4a), and the amount of quaternary amine increases apparently (about 31.29\%, Fig. 4c). This result indicates that more PDPAEMA molecules are protonated. When the surface is further treated with the basic solution, the protonated PDPAEMA returns to its deprotonated state. The $\mathrm{Cl} 2 \mathrm{p}$ peak disappears (red line in Fig. 4a), and the intensity of the peak ascribed to the quaternary amine decreases to the original state (i.e., the amount of the quaternary amine decreases to about $6.5 \%$, Fig. $4 \mathrm{~d}$ ). Therefore, after modification with PDPAEMA, the surface has $\mathrm{pH}$ responsivity, and the protonated and deprotonated states of PDPAEMA can reversibly appear as the water $\mathrm{pH}$ changes.

The inner mechanism is carefully analyzed to further understand the excellent controllability of wettability. The result suggests that the great shape memory property of SMP (Fig. 5a, b) and the pH responsivity of PDPAEMA (Fig. 5c, d) can be attributed to the synergistic effect of the tunable surface microstructures and chemical compositions, respectively. As reported, two structural requirements, namely, invertible thermal exchange for temporary shape fixing/recovery and crosslinking network, are necessary to obtain an excellent shape memory effect for polymers [53]. In our work, the obtained polymer has a good ability of thermal invertible phase exchange (Fig. S6b) and has a cross-linked network (i.e., the tail-to-tail correlations of alkyl chains form physical crosslinking, and the reaction of $-\mathrm{NH}_{2}$ and epoxy groups forms chemical crosslinking) [54]. Therefore, the asprepared materials exhibit a great shape memory ability, with about $99 \%$ of shape recovery ratio (Fig. S13). As such, the material shows and memorizes various micromorphologies. In Fig. 5a, the obtained surface has a permanent shape of upright pillar structures (left picture in Fig. 5a). The molecular chains have the highest entropy (right picture in Fig. 5a), and they reside in a thermodynamically stable state [55]. The surface is heated to a 

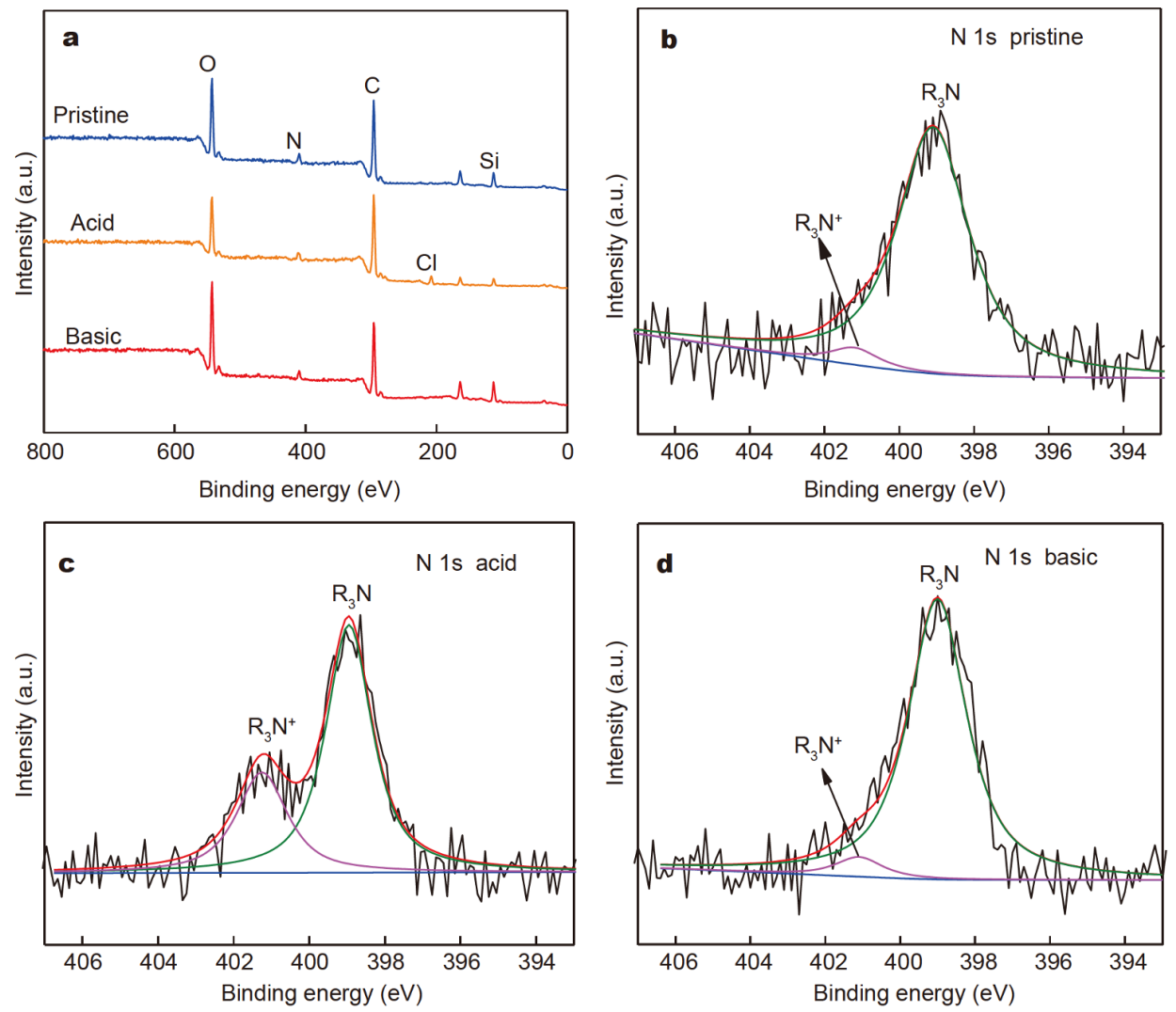

Figure 4 XPS survey spectra. (a) PDPAEMA-modified surface in different states: blue, orange, and red lines represent the pristine state, after acid treatment, and after base treatment, respectively. (b) High-resolution N 1s spectrum of pristine PDPAEMA-modified surface, (c) after acid treatment, and (d) after base treatment. The results confirm an excellent $\mathrm{pH}$ responsivity of the prepared surface.

temperature higher than $T_{\mathrm{g}}$ to change the surface microstructure, which consequently softens. Under this condition, the surface pillars collapse when they are pressed under an external force (left picture in Fig. 5b). In this process, the crosslinking network can prevent the long-range chain from slipping; as a result, some entropy can be trapped, and a new molecular chain conformation can be formed (right picture in Fig. 5b) [53]. When the surface is cooled to room temperature under the same external force, the frozen molecular chain segment retains the pillars' shape in a collapsed state. Therefore, a temporarily deformed shape can be achieved. Furthermore, as the surface is reheated without the external force (above the $T_{\mathrm{g}}$ ), the mobility of the molecular chains of SMP can be reactivated. The favorable tendency for an increase in entropy thermodynamically allows the molecular chains to recover their permanent conformation. Thus, the original upright state of the collapsed pillars can be restored (Fig. 3), and a reversible transition between upright and collapsed states can be repeatedly controlled by cycled pressing/recovery processes.
This description clearly explains the variation in the shape of the pillar on the surface. When the pillars are in an upright state, for an acid droplet that contacts the surface, the grafted PDPAEMA is protonated (Fig. 5c) and shows hydrophilicity (Fig. 5e); PDPAEMA exhibits hydrophilicity and hydrophobicity after it is treated with water solutions with $\mathrm{pH}$ lower or higher than its $\mathrm{pKa}$ (about 6.2 [56]) because of the protonation and deprotonation of PDPAEMA, respectively. According to the Wenzel equation [57], the hydrophilicity of surfaces can be strengthened when surface roughness increases. The strong 3D capillary effect of the upright pillars can cause water to enter the pillar structures (inset in Fig. 5e). Therefore, the surface displays the superhydrophilicity of water with $\mathrm{pH} 2$ (Fig. 1l). When a basic water droplet is used $(\mathrm{pH}>\mathrm{pKa})$, PDPAEMA becomes deprotonated and shows hydrophobicity (Fig. 5d, f). On the occasion, air is trapped in the microstructure with the aid of upright pillars, and the droplet exists in a composited Cassie state (inset in Fig. 5f) [58]. In this case, the trapped air helps reduce the solid/liquid contact area. Consequently, the 


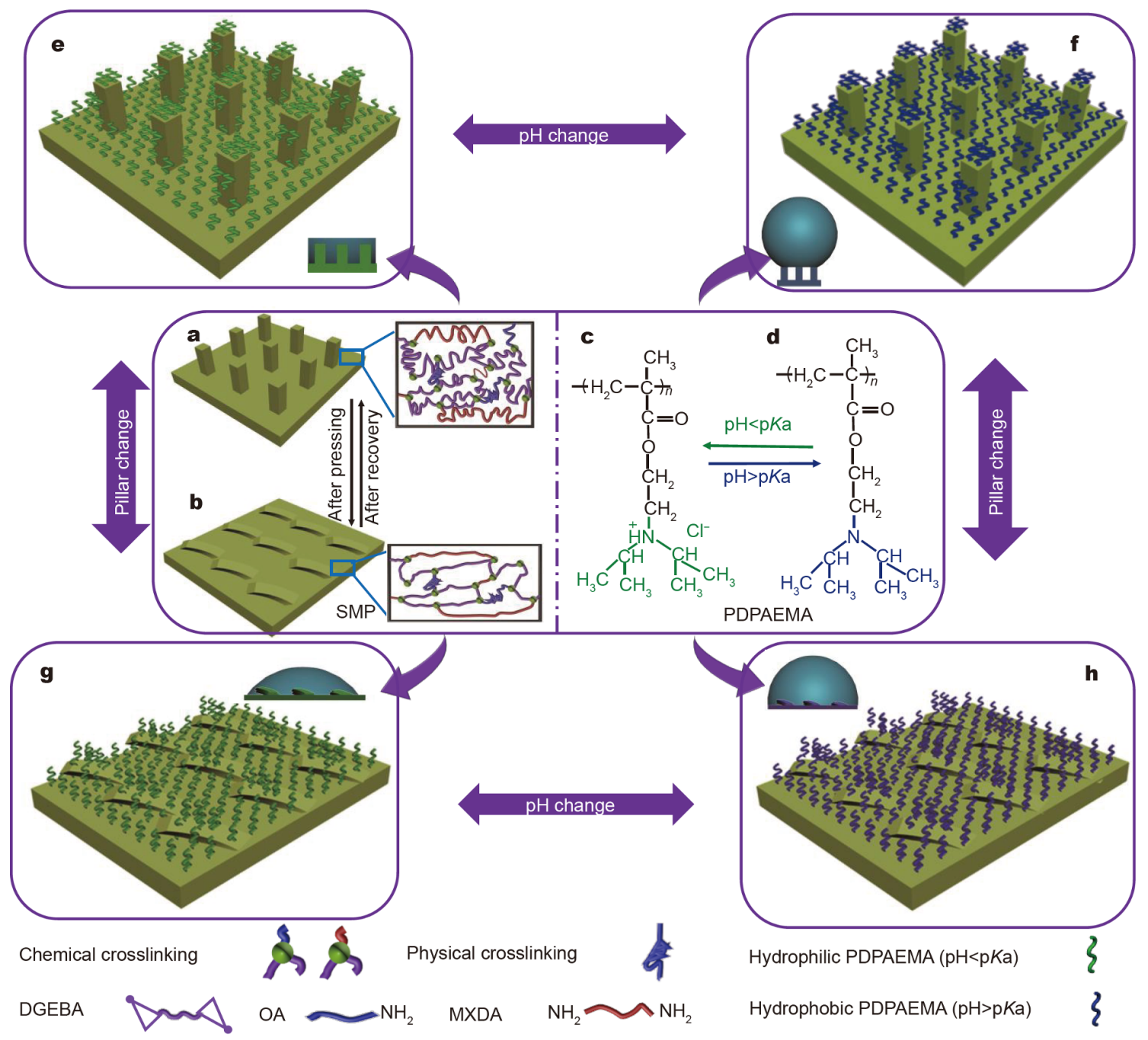

Figure 5 Schematic of the responsive mechanisms of SMP, PDPAEMA, and different water/solid contact models. Numerous crosslinking networks are contained in the obtained SMP substrate, and the highest entropy exists in the conformation of the molecular chains (inset in (a)), and the straight pillars are the permanent structure. Under this condition, when an acidic water droplet contacts the surface, PDPAEMA becomes protonated and hydrophilic ( $\mathrm{pH}<\mathrm{pKa}$, in (c)); under the 3D capillary action, the water droplet enters the microstructure, and the surface shows superhydrophilicity (e). On the contrary, when a basic water droplet is used, PDPAEMA becomes deprotonated and hydrophobic ( $\mathrm{pH}>\mathrm{pKa}$, in (d)); the droplet resides in a composite Cassie state because a layer of air can be trapped in the microstructure, which can effectively provide the surface with superhydrophobicity (f). When the surface is heated to a temperature higher than $T_{\mathrm{g}}$ and pressed under an external force, the crosslinking network can prevent a long-range chain slippage, resulting in the establishment of a new chain conformation containing a low entropy (inset in (b)) and a collapsed pillar structure. On a surface with collapsed pillars, the enhanced effect of the microstructure on wettability is reduced; thus, the surface shows general hydrophilicity $(\mathrm{g})$ and hydrophobicity $(\mathrm{h})$ for acidic and basic droplets, respectively. When the surface is reheated at $>T_{\mathrm{g}}$, the mobility of molecular chains can be reactivated, and the SMP molecular chain conformation can be recovered after the recovery of the surface structure because of the thermodynamically favorable tendency for increasing entropy. As a result, a reversible exchange between superhydrophobicity and superhydrophilicity can be observed. Surface microstructure and chemistry are stimulus-responsive, so numerous degrees of wetting control can be realized by regulating the two factors.

surface presents superhydrophobicity for a basic droplet (Fig. 11). The collapse of the surface microstructure can result in a decrease in surface roughness and ultimately reduce the enhanced effect on surface wettability [59-61]. Therefore, the surfaces with such collapsed pillars show hydrophilicity for acidic water (Fig. 5g) and hydrophobicity for basic water (Fig. 5h). PDPAEMA is stable when the temperature is $<200^{\circ} \mathrm{C}$ (Fig. S14), indicating that the heating temperature used for microstructural variation does not affect PDPAEMA. The influence of the 
physical deformation of pillars on surface chemistry is negligible (Fig. S15 and Table S1). As such, when the structure of pillars is recovered, a reversible exchange from superhydrophilicity to superhydrophobicity can be observed again. Surface chemical compositions and microstructures can be reversibly and precisely manipulated separately or synergistically; hence, a reversible exchange among multiple wettabilities and the precise control of surface wettability can be achieved (Fig. 1l, m).

In this work, the surface with precise controllability in wettability shows potential for many applications, such as platforms for producing gradient wetting. Materials with precise gradient wettability from superhydrophobicity to superhydrophilicity have been widely explored because of some particular applications, such as accurate drug release [14] and precise oil-water separation [11]. In our work, precise gradient wetting can be achieved on our surface because of excellent controllability. The surface is divided into six parts from left to right by controlling the pillar state and the $\mathrm{pH}$ of droplets to demonstrate this concept: upright pillars with $\mathrm{pH} 2$, upright pillars with $\mathrm{pH}$ 6 , collapsed pillars with $\mathrm{pH} 6$, collapsed pillars with $\mathrm{pH} 8$, upright pillars with $\mathrm{pH} 8$, and upright pillars with $\mathrm{pH} 12$ (Fig. 6a). Interestingly, the designed surface can exhibit six wetting states with CAs of $0^{\circ}, 40^{\circ}, 55^{\circ}, 83^{\circ}, 120^{\circ}$, and $150^{\circ}$ (Fig. 6b, from the left to right), indicating that gradient wetting from superhydrophilicity to the superhydrophobicity can be realized on the surface. In addition to the application in creating a gradient surface, many other fields, such as unidirectional droplet transportation and fog harvesting, can benefit from surfaces with precise wetting controllability.

\section{CONCLUSION}

In summary, a dual-stimulus-responsive strategy for smart and accurate wetting control is developed by cooperatively adjusting the chemical compositions and microstructures of surfaces. As a demonstration, a surface is obtained by modifying the pillar-structured SMP with pH-responsive PDPAEMA. On the prepared surface, different wettabilities between superhydrophilicity and superhydrophobicity with CA differences of less than $15^{\circ}$ can be accurately and reversibly manipulated. The samples exhibit an excellent controllability because of the combined effect of the tunable surface chemical compositions and microstructures, which originate from the $\mathrm{pH}$ responsivity of PDPAEMA and the shape memory ability of SMP, respectively. Additionally, the prepared sample can be used as a platform to create gradient wettings. This paper reports a surface wetting control strategy, which

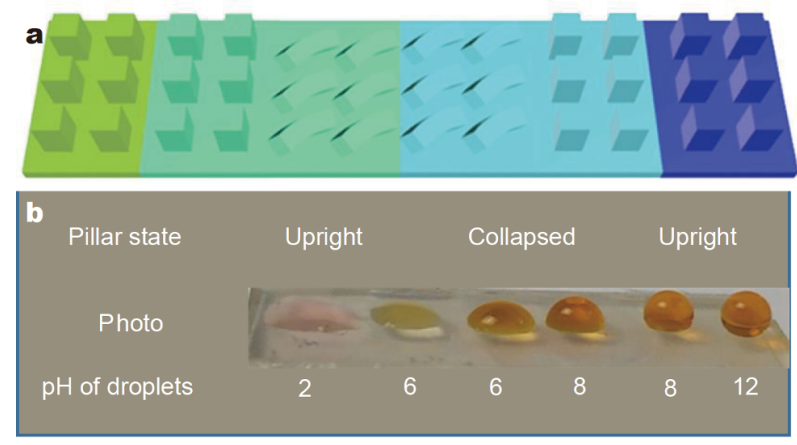

Figure 6 Application of the surface as a platform for creating gradient wettings from superhydrophilicity to superhydrophobicity based on precise controllability. (a) Illustration of the pillars designed as follows from left to right: upright pillars with $\mathrm{pH} \mathrm{2}$, upright pillars with $\mathrm{pH} 6$, collapsed pillars with $\mathrm{pH} 6$, collapsed pillars with $\mathrm{pH} 8$, upright pillars with $\mathrm{pH} 8$, and upright pillars with $\mathrm{pH}$ 12. (b) Gradient wetting performance from superhydrophilicity to superhydrophobicity can be obtained on the surface.

not only provides some novel ideas for designing functional surfaces with tunable wettability but also overcomes the drawbacks of current precise wetting control methods. With the special advantage of the prepared surface, the proposed strategy shows potential for various applications, including controllable drug delivery, friction, and catalysis. Furthermore, given the design simplicity, this strategy can be easily extended to develop and fabricate other similar functional materials by combining SMP with other smart molecules responsive to light, temperature, electricity, magnetic field, and other stimuli.

Received 7 September 2020; accepted 2 November 2020;

published online 2 February 2021

1 Lu Y, Sathasivam S, Song J, et al. Robust self-cleaning surfaces that function when exposed to either air or oil. Science, 2015, 347: 1132-1135

2 Wang S, Liu K, Yao X, et al. Bioinspired surfaces with superwettability: New insight on theory, design, and applications. Chem Rev, 2015, 115: 8230-8293

3 Lou X, Huang Y, Yang X, et al. External stimuli responsive liquidinfused surfaces switching between slippery and nonslippery states: Fabrications and applications. Adv Funct Mater, 2020, 30: 1901130

4 Zhang $\mathrm{H}$, Lai $\mathrm{H}$, Cheng $\mathrm{Z}$, et al. In-situ switchable superhydrophobic shape memory microstructure patterns with reversible wettability and adhesion. Appl Surf Sci, 2020, 525: 146525

5 Wang Z, Zhang S, Gao S, et al. A simple, low-cost method to fabricate drag-reducing coatings on a macroscopic model ship. Chem Res Chin Univ, 2018, 34: 616-621

6 Chen L, Liu M, Bai H, et al. Antiplatelet and thermally responsive poly $(N$-isopropylacrylamide) surface with nanoscale topography. J Am Chem Soc, 2009, 131: 10467-10472

7 Sun M, Feng W, Wang B, et al. Studies on surface properties and cell adhesion properties of BSA modified DBM scaffold. Chem Res Chin Univ, 2019, 35: 700-707 
8 Sheparovych R, Motornov M, Minko S. Low adhesive surfaces that adapt to changing environments. Adv Mater, 2009, 21: 1840-1844

9 Li C, Yu C, Hao D, et al. Smart liquid transport on dual biomimetic surface via temperature fluctuation control. Adv Funct Mater, 2018, 28: 1707490

10 Han K, Heng L, Zhang Y, et al. Slippery surface based on photoelectric responsive nanoporous composites with optimal wettability region for droplets' multifunctional manipulation. Adv Sci, 2019, 6: 1801231

11 Wang B, Liang W, Guo Z, et al. Biomimetic super-lyophobic and super-lyophilic materials applied for oil/water separation: A new strategy beyond nature. Chem Soc Rev, 2015, 44: 336-361

12 Zhang J, Zhang F, Song J, et al. Electrospun flexible nanofibrous membranes for oil/water separation. J Mater Chem A, 2019, 7: 20075-20102

13 Leon AC, Imperial RES, Chen Q, et al. One-step fabrication of superhydrophobic/superoleophilic electrodeposited polythiophene for oil and water separation. Macromol Mater Eng, 2019, 304: 1800722

14 Yohe ST, Colson YL, Grinstaff MW. Superhydrophobic materials for tunable drug release: Using displacement of air to control delivery rates. J Am Chem Soc, 2012, 134: 2016-2019

15 Sun T, Wang G, Feng L, et al. Reversible switching between superhydrophilicity and superhydrophobicity. Angew Chem Int Ed, 2004, 43: 357-360

16 Cheng M, Liu Q, Ju G, et al. Bell-shaped superhydrophilicsuperhydrophobic-superhydrophilic double transformation on a pH-responsive smart surface. Adv Mater, 2014, 26: 306-310

17 Xiao M, Guo X, Cheng M, et al. $\mathrm{pH}$-responsive on-off motion of a superhydrophobic boat: Towards the design of a minirobot. Small, 2014, 10: 859-865

18 Hong X, Gao X, Jiang L. Application of superhydrophobic surface with high adhesive force in no lost transport of superparamagnetic microdroplet. J Am Chem Soc, 2007, 129: 1478-1479

19 Lim HS, Lee SG, Lee DH, et al. Superhydrophobic to superhydrophilic wetting transition with programmable ion-pairing interaction. Adv Mater, 2008, 20: 4438-4441

20 Zhu W, Zhai J, Sun Z, et al. Ammonia responsive surface wettability switched on indium hydroxide films with micro- and nanostructures. J Phys Chem C, 2008, 112: 8338-8342

21 Minko S, Müller M, Motornov M, et al. Two-level structured selfadaptive surfaces with reversibly tunable properties. J Am Chem Soc, 2003, 125: 3896-3900

22 Seo J, Lee JS, Lee K, et al. Switchable water-adhesive, superhydrophobic palladium-layered silicon nanowires potentiate the angiogenic efficacy of human stem cell spheroids. Adv Mater, 2014, 26: 7043-7050

23 Krupenkin TN, Taylor JA, Wang EN, et al. Reversible wettingdewetting transitions on electrically tunable superhydrophobic nanostructured surfaces. Langmuir, 2007, 23: 9128-9133

24 Lahann J, Mitragotri S, Tran TN, et al. A reversibly switching surface. Science, 2003, 299: 371-374

25 Gao W, Wang J, Zhang X, et al. Electric-tunable wettability on a paraffin-infused slippery pattern surface. Chem Eng J, 2020, 381: 122612

26 Qu R, Zhang W, Li X, et al. A smart nano- $\mathrm{V}_{2} \mathrm{O}_{5} / \mathrm{ODA}$-coated mesh for a co-responsive photo-induced wettability transition and ROS generation for in situ water purification. J Mater Chem A, 2018, 6: 18003-18009

27 Caputo G, Cortese B, Nobile C, et al. Reversibly light-switchable wettability of hybrid organic/inorganic surfaces with dual micro-/ nanoscale roughness. Adv Funct Mater, 2009, 19: 1149-1157

28 Zong $\mathrm{C}, \mathrm{Hu} \mathrm{M}$, Azhar U, et al. Smart copolymer-functionalized flexible surfaces with photoswitchable wettability: From superhydrophobicity with "rose petal" effect to superhydrophilicity. ACS Appl Mater Interfaces, 2019, 11: 25436-25444

29 Tian D, Zhang N, Zheng X, et al. Fast responsive and controllable liquid transport on a magnetic fluid/nanoarray composite interface. ACS Nano, 2016, 10: 6220-6226

30 Grigoryev A, Tokarev I, Kornev KG, et al. Superomniphobic magnetic microtextures with remote wetting control. J Am Chem Soc, 2012, 134: 12916-12919

31 Zhao S, Xia H, Wu D, et al. Mechanical stretch for tunable wetting from topological PDMS film. Soft Matter, 2013, 9: 4236-4240

32 Lee SG, Lee DY, Lim HS, et al. Switchable transparency and wetting of elastomeric smart windows. Adv Mater, 2010, 22: 50135017

$33 \mathrm{Wu} \mathrm{D}, \mathrm{Wu} \mathrm{SZ}$, Chen QD, et al. Curvature-driven reversible in situ switching between pinned and roll-down superhydrophobic states for water droplet transportation. Adv Mater, 2011, 23: 545-549

34 Zhu Y, Antao DS, Xiao R, et al. Real-time manipulation with magnetically tunable structures. Adv Mater, 2014, 26: 6442-6446

35 Chen CM, Yang S. Directed water shedding on high-aspect-ratio shape memory polymer micropillar arrays. Adv Mater, 2014, 26 : 1283-1288

36 Wang L, Zhao Y, Tian Y, et al. A general strategy for the separation of immiscible organic liquids by manipulating the surface tensions of nanofibrous membranes. Angew Chem Int Ed, 2015, 54: 1473214737

37 Li G, Hong G, Dong D, et al. Multiresponsive graphene-aerogeldirected phase-change smart fibers. Adv Mater, 2018, 30: 1801754

38 Jiang Y, Wan P, Smet M, et al. Self-assembled monolayers of a malachite green derivative: Surfaces with $\mathrm{pH}$ - and UV-responsive wetting properties. Adv Mater, 2008, 20: 1972-1977

39 Wang R, Xie T. Shape memory- and hydrogen bonding-based strong reversible adhesive system. Langmuir, 2010, 26: 2999-3002

40 Sarwate P, Chakraborty A, Garg V, et al. Controllable strain recovery of shape memory polystyrene to achieve superhydrophobicity with tunable adhesion. J Micromech Microeng, 2014, 24: 115006

41 Schauer S, Meier T, Reinhard M, et al. Tunable diffractive optical elements based on shape-memory polymers fabricated via hot embossing. ACS Appl Mater Interfaces, 2016, 8: 9423-9430

$42 \mathrm{Xu} \mathrm{H}, \mathrm{Yu} \mathrm{C}$, Wang S, et al. Deformable, programmable, and shapememorizing micro-optics. Adv Funct Mater, 2013, 23: 3299-3306

43 Ramaraju H, Akman RE, Safranski DL, et al. Designing biodegradable shape memory polymers for tissue repair. Adv Funct Mater, 2020, 30: 2002014

44 Zhao L, Zhao J, Liu Y, et al. Continuously tunable wettability by using surface patterned shape memory polymers with giant deformability. Small, 2016, 12: 3327-3333

45 Shahsavan H, Salili SM, Jákli A, et al. Smart muscle-driven selfcleaning of biomimetic microstructures from liquid crystal elastomers. Adv Mater, 2015, 27: 6828-6833

46 Wang W, Salazar J, Vahabi H, et al. Metamorphic superomniphobic surfaces. Adv Mater, 2017, 29: 1700295

47 Lv T, Cheng Z, Zhang E, et al. Self-restoration of superhydrophobicity on shape memory polymer arrays with both crushed microstructure and damaged surface chemistry. Small, 2017, 13: 1503402 
48 Lv T, Cheng Z, Zhang D, et al. Superhydrophobic surface with shape memory micro/nanostructure and its application in rewritable chip for droplet storage. ACS Nano, 2016, 10: 9379-9386

49 Stratakis E, Mateescu A, Barberoglou M, et al. From superhydrophobicity and water repellency to superhydrophilicity: Smart polymer-functionalized surfaces. Chem Commun, 2010, 46: 41364138

50 Feng L, Li S, Li Y, et al. Super-hydrophobic surfaces: From natural to artificial. Adv Mater, 2002, 14: 1857-1860

51 Zhao T, Nie FQ, Jiang L. Precise control of wettability from LCST tunable surface. J Mater Chem, 2010, 20: 2176-2181

52 Dang Z, Liu L, Li Y, et al. In situ and ex situ $\mathrm{pH}$-responsive coatings with switchable wettability for controllable oil/water separation. ACS Appl Mater Interfaces, 2016, 8: 31281-31288

53 Xie T. Recent advances in polymer shape memory. Polymer, 2011, 52: 4985-5000

54 Puig J, Zucchi IA, Hoppe CE, et al. Epoxy networks with physical cross-links produced by tail-to-tail associations of alkyl chains. Macromolecules, 2009, 42: 9344-9350

55 Zhao Q, Qi HJ, Xie T. Recent progress in shape memory polymer: New behavior, enabling materials, and mechanistic understanding. Prog Polym Sci, 2015, 49-50: 79-120

56 Lee CH, Kang SK, Lim JA, et al. Electrospun smart fabrics that display pH-responsive tunable wettability. Soft Matter, 2012, 8: 10238-10240

57 Wenzel RN. Resistance of solid surfaces to wetting by water. Ind Eng Chem, 1936, 28: 988-994

58 Cassie ABD, Baxter S. Wettability of porous surfaces. Trans Faraday Soc, 1944, 40: 546-551

59 Bellanger H, Darmanin T, Taffin de Givenchy E, et al. Chemical and physical pathways for the preparation of superoleophobic surfaces and related wetting theories. Chem Rev, 2014, 114: 26942716

60 Lafuma A, Quéré D. Superhydrophobic states. Nat Mater, 2003, 2: 457-460

61 Liu M, Wang S, Jiang L. Nature-inspired superwettability systems. Nat Rev Mater, 2017, 2: 17036

Acknowledgements This work was supported by the National Natural Science Foundation of China (21674030, 22075061 and 51790502), the Funding of Key Laboratory of Bioinspired Materials and Interfacial Science, the Technical Institute of Physics and Chemistry, Chinese Academy of Sciences, and China National Postdoctoral Program for Innovative Talents (BX20200106).

Author contributions Liu Y and Cheng $\mathrm{Z}$ conceived the idea; Jiang L revised the idea; Zhang D prepared the materials and conducted most of the measurements; Zhang D and Xia Q wrote the paper; Lai H revised the paper; Liu P performed most of the SEM characterizations; Zhang $\mathrm{H}$ analyzed the XPS results. All authors discussed the results and commented on the manuscript.

Conflict of interest The authors declare that they have no conflict of interest.

Supplementary information Experimental details and supporting data are available in the online version of the paper.

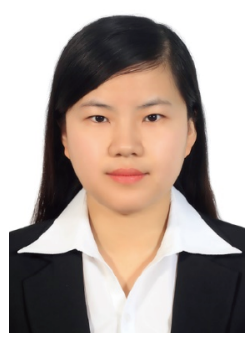

Dongjie Zhang received her BS degree (2014) and $\mathrm{PhD}$ degree (2020) at Harbin Institute of Technology, China. During 2018 to 2019, she stayed at the University of British Columbia in Canada as a visiting student in Mark MacLachlan's group. She is currently a postdoc at the School of Chemistry and Chemical Engineering, Harbin Institute of Technology, Harbin, China. Her present research interest focuses on shape memory polymer with special wettability.

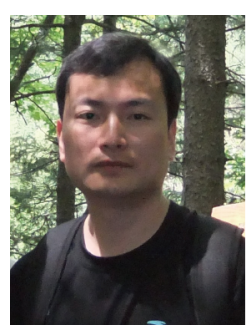

Zhongjun Cheng obtained his BS degree (2003) and MS degree (2006) in chemistry at Jilin University in Jilin, China, and $\mathrm{PhD}$ degree (2009) at the Institute of Chemisty, Chinese Academy of Sciences under the supervision of Prof. Lei Jiang. $\mathrm{He}$ is currently an associate professor at Harbin Institute of Technology, Harbin, China. His scientific interest is design and fabrication of superwetting materials with dynamic tunable micro-/nano-structures, and related applications.

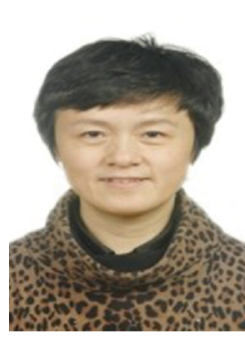

Yuyan Liu obtained her BS and PhD degrees from the Department of Polymer Materials and Engineering, Harbin Institute of Technology, Harbin, China. During 2001 to 2002, she worked at the University of Tokyo in Japan as a visiting scholar. She is currently a professor at Harbin Institute of Technology. Her research interests include construction and intelligent control of micro-/nano-structure on polymer surface, spatial flexible rigid materials, shape memory polymer and composite materials, and recycling of polymer materials.

\section{具有智能、精细多浸润状态调控的双响应形状记 忆聚合物阵列}

张东杰 ${ }^{1}$, 夏琦兴 ${ }^{2}$, 来华 ${ }^{1}$, 成中军 ${ }^{1^{*}}$, 刘鹏昌 ${ }^{1}$, 张海洋 ${ }^{1}$, 刘宇艳 ${ }^{*}$, 江雷 $^{3}$

摘要 近年来, 具有超亲水到超疏水转变的智能可控浸润性表面引 起了人们的广泛关注. 由于大多数报道仅采用单一的调控手段, 即 单一表面化学调控或单一的微结构调控, 表面浸润性的智能、精 细调控目前仍然很难实现. 本文中, 我们将 $\mathrm{pH}$ 响应的分子聚2-(二 异丙基氨基)甲基丙烯酸乙酯(PDPAEMA)修饰到温度响应的形状 记忆聚合物(SMP)阵列表面, 获得了一种可实现表面化学和微结构 协同调控的双刺激响应材料. 其中PDPAEMA ( $\mathrm{pH}$ 刺激)和SMP (温 度刺激)分别保证了表面化学和微结构的可调节性, 通过调节 $\mathrm{pH}$ 和 温度, 可使表面化学和表面微结构协同作用, 从而在所得表面上实 现超亲水到超疏水范围的智能可逆转变, 转变精度小于 $15^{\circ}$. 另外, 利用其优异的浸润性可控特性, 所得表面可用于制备梯度浸润性 控制平台. 本文所制备表面在自适应液体微透镜、精确药物释 放、选择性催化等领域具有良好的应用前景, 同时也为设计和开 发新型智能浸润性提供了参考. 